\title{
CMI: An Online Multi-objective Genetic Autoscaler for Scientific and Engineering Workflows in Cloud Infrastructures with Unreliable Virtual Machines
}

\author{
David A. Monge ${ }^{\mathrm{a}, *}$, Elina Pacini ${ }^{\mathrm{a}, \mathrm{b}}$, Cristian Mateos $^{\mathrm{c}}$, Enrique Alba $^{\mathrm{d}}$, Carlos García Garino ${ }^{\mathrm{a}}$ \\ ${ }^{a}$ ITIC, Universidad Nacional de Cuyo. Mendoza, Argentina \\ ${ }^{b}$ Consejo Nacional de Investigaciones Científicas y Técnicas (CONICET). Argentina. \\ ${ }^{c}$ ISISTAN-UNICEN-CONICET. Tandil, Buenos Aires, Argentina. \\ ${ }^{d}$ Departamento de Lenguajes y Ciencias de la Computación, Universidad de Málaga. Spain.
}

\begin{abstract}
Cloud Computing is becoming the leading paradigm for executing scientific and engineering workflows. The large-scale nature of the experiments they model and their variable workloads make clouds the ideal execution environment due to prompt and elastic access to huge amounts of computing resources. Autoscalers are middleware-level software components that allow scaling up and down the computing platform by acquiring or terminating virtual machines (VM) at the time that workflow's tasks are being scheduled. In this work we propose a novel online multi-objective autoscaler for workflows denominated Cloud Multi-objective Intelligence (CMI), that aims at the minimization of makespan, monetary cost and the potential impact of errors derived from unreliable VMs. In addition, this problem is subject to monetary budget constraints. CMI is responsible for periodically solving the autoscaling problems encountered along the execution of a workflow. Simulation experiments on four well-known workflows exhibit that CMI significantly outperforms a state-of-the-art autoscaler of similar characteristics called Spot Instances Aware Autoscaling (SIAA). These results convey a solid base for deepening in the study of other meta-heuristic methods for autoscaling workflow applications using cheap but unreliable infrastructures.
\end{abstract}

Keywords: Cloud Computing, Autoscaling, Scientific workflow, Multi-objective optimization, Evolutionary Algorithm, Unreliable VM Instance

\section{Introduction}

Today's scientific workflows require large amounts of computational resources to satisfy the resourceintensive nature of the experiments they model. A scientific workflow comprises a series of software components denominated tasks, which have dependencies between them, and impose a partial execution order of tasks. Scientific workflows are useful for modeling a large number of engineering problems in areas such as Astronomy [1], and Environmental Engineering [2]. The conjunction of such structure of dependencies and the heterogeneity of tasks determine that, during the execution of the workflow, workload can notably vary, which is a scenario suitable for resource elasticity capabilities of cloud infrastructures [3].

Autoscalers exploit such elasticity capabilities by dynamically adapting the cloud infrastructure according to the computational workflow requirements. At the same time, autoscaling must be performed having in mind some optimization criteria such as execution time, monetary cost or other aspects of the workflow execution. To such end, autoscalers acquire or terminate virtual machines (VMs) and schedule workflow tasks onto such VMs.

\footnotetext{
${ }^{*}$ Corresponding author.

Email addresses: dmonge@uncu.edu.ar (David A. Monge), epacini@uncu.edu.ar (Elina Pacini), cmateos@conicet.gov.ar (Cristian Mateos), eat@lcc.uma.es (Enrique Alba), cgarcia@itu.uncu.edu.ar (Carlos García Garino)
} 
To avoid ambiguity, through the remainder of this article we will adopt the Amazon Elastic Compute Cloud (EC2) nomenclature and we will refer to VMs as instances. Instances can be classified according to: (a) their type, which determines a specific preset of hardware and software configurations 1 , and (b) the pricing model adopted, i.e., the on-demand and the spots models. Instance types determine different computing capabilities and configurations such as the number of CPUs, CPU speed, memory size, operating system, etc. These characteristics determine the processing time of executed tasks, in other words, they rule tasks' performance.

On the other hand, the pricing model determines the cost of instances and their behavior. Instances acquired under the spots mode ${ }^{2}$, are usually cheaper than instances acquired under the alternative on-demand pricing model. However, instances under the spots model are unreliable as they can fail, thus abruptly terminating the execution of any running task assigned to the instance. These failures can potentially impact on workflow execution time (i.e. makespan) and monetary cost.

Workflow autoscaling consider such prone-to-failure instances are a very challenging problem that implies a compromise between the objectives of makespan, monetary cost and reliability. In other words, this is a multi-objective minimization problem. In a previous work of our own [3] we tackled scientific workflow autoscaling using spot instances by solving a makespan minimization problem subject to budget constraints (i.e. limiting the maximum cost). To cope with spot instances unreliability, tasks were heuristically scheduled with the aim of mitigating the negative effects of out-of-bid (OOB) errors on the workflow makespan. Such autoscaler is called Spot Instances Aware Autoscaling (SIAA).

In other of our works [4] we addressed the multi-objective minimization problem aiming at reducing makespan, monetary cost and the potential impact of OOB errors as stated in the previous paragraphs. However, in such article we focused on a different type of scientific application called parameter sweep experiments (PSEs). Just like workflows, PSEs are applications that comprise a set of tasks to realize a given experiment, but in PSEs' tasks are independent (i.e. there are no inter-task dependencies) and mostly homogeneous [5], which determines a completely different and simpler autoscaling scenario. Such autoscaler is called Multi-objective Evolutionary Autoscaler (MOEA) to solve the mentioned problem.

To the best of our knowledge, most cloud autoscaling strategies have been proposed for the efficient management of workflow applications mainly subject to deadline constraints [6, 7, 8]. Moreover, from the revised literature, we have not found works where the authors minimize the potential impact of OOB errors along with minimizing both makespan and monetary cost of scientific workflows in an online scenario as we propose in this work.

Concretely, the key idea of this work is to propose a new autoscaler to solve the autoscaling of scientific workflows for online scenarios, i.e., scenarios where the availability of the virtual infrastructure varies constantly throughout the applications execution. Motivated by this idea, this article brings in the following contributions:

- A novel online multi-objective autoscaler and a major extension to the mathematical formulation of the autoscaler proposed in [4] for executing scientific workflows. We call this autoscaler Cloud Multi-objective Intelligence (CMI). CMI is an autoscaler for scientific workflows that considers spot instances and minimizes makespan, cost and the potential impact of OOB errors. The mathematical formulation of the problem and a description of the CMI autoscaler are given in Section 2

- The proper parameterization values for the optimization algorithm used by CMI considering four real large-scale scientific workflows derived from the Astronomy and Environmental Engineering [9, 1] domains. Tasks execution data extracted from such workflows, actual characteristics of the Amazon EC2 instances used, on-demand prices, and data of spot prices are discussed in Section 3 Then, Section 4 presents a sensitivity analysis performed to obtain the best hyperparameter sets for the genetic part of CMI.

- An experimental validation demonstrating that CMI outperforms SIAA [3], a recently published state-of-the-art autoscaler for workflow applications w.r.t. relevant metrics, particularly makespan,

\footnotetext{
${ }^{1} \mathrm{EC} 2$ currently offers nearly 60 different instance types.

${ }^{2}$ Amazon EC2 spot instances: http://aws.amazon.com/ec2/spot//[Online; accessed September-2018]
} 
economic cost, and $L_{2}$-norm of such metrics. As CMI, SIAA also considers spot instances, but in contra-position, SIAA is a heuristic-based method rather than a meta-heuristic one. In our experiments, SIAA was parameterized to operate under different configurations to settle the basis for a rigorous comparison. Section 5 discusses the mentioned experiments and presents the results of statistical significance tests that back up the strength of our claims.

Finally, Section 6 surveys relevant related work and Section 7 concludes this work and discusses future prospective extensions.

\section{Online Multi-objective Workflow Autoscaling}

This work deals with the problem of autoscaling scientific and engineering workflows in public clouds like the Amazon EC2. Autoscaling workflows requires the acquisition of instances that might belong to different types and they can be purchased under different pricing models, in order to optimize a given criteria. In this work, we propose to minimize workflow makespan, monetary cost and probability of outof-bid (OOB) errors. These characteristics make the problem very complex since it has many possible solutions.

In practice, when a scientific workflow is executed, tasks' performance vary across different instance types where each type defines specific characteristics of the instances to acquire such as processing power, number of processors, memory size, memory speed, disk size, operating system, etc.

In this work, we assume that instances are charged by hour under two possible pricing models namely the on-demand model and the spots model. The on-demand pricing model offers the access to reliable instances at a fixed price charged by hour of computation. Instances acquired under this pricing model are usually more expensive than instances acquired using the spots model. For simplicity, we will refer to instances acquired under the on-demand pricing model as on-demand instances.

Alternatively, under the spots pricing model, instance's prices fluctuate over time and are usually much lower than the prices of the on-demand model. To request an instance under the spots model, the user must bid the maximum price he/she is willing to pay, and while the actual price is below the user's bid, the instance will remain operative. However, if the actual spot price overcomes the user's bid, an OOB error occurs forcing the termination of the affected instance and thus the execution of all of the tasks that might be running on it. Thus, we say that these kind of instances are unreliable. We will refer such instances as spot instances.

It is worth to remark that spot-instance prices vary in an unpredictable way, therefore the occurrence of OOB errors is bound to the bid price selected. Moreover, considering the large scale of scientific workflows, the extent of the potential impact of OOB errors depends also on the amount of spot instances running. For such reason, during the execution of a scientific workflow, decisions involving spot instances are very important as they compromise execution makespan, monetary cost and reliability. For example, lower bids allow to reduce execution costs but at the same time they increase the probability that an OOB error occurs, which negatively affects makespan. Conversely, higher bids reduce the probability of OOB errors, which helps in preserving makespan but they increase the overall cost of executing a workflow. Therefore, it is for these reasons that we are dealing with an online multi-objective problem where it is important to minimize the occurrence of OOB errors, makespan, and cost [4]. This is a complex problem and it has not been previously addressed with the same approach adopted in this work.

Concretely, our autoscaling strategy, called Cloud Multi-objective Intelligence (CMI), aims at determining the proper amount of instances for each combination of instance type and pricing model, and the adequate bid for spot instances needed for the workflow at hand. The objective of CMI is to obtain the proper virtual infrastructure configuration for minimizing the makespan, cost and the potential impact of OOB errors on the execution of workflow tasks. Moreover, it is important to mention that autoscaling accounts two interrelated problems:

${ }^{3}$ A complete list of the different instance types offered by Amazon EC2 is available at https://aws .amazon.com/ec2/ instance-types/ 
1. determining a scaling plan $X$ that describes the adequate virtual infrastructure setting to request to the cloud provider, i.e. the amount of instances for each combination of instance type and pricing model, and the bid price for the accounted spot instances; and

2. scheduling the workflow's tasks onto the instances acquired according to the scaling plan.

Both of these problems are NP-hard, and therefore many researchers have proposed heuristic and metaheuristic solutions [10, 3, 4]. Remarkably, our proposed CMI autoscaler searches for solutions which include the bid price for the spot instances chosen instead of requiring a particular spot prices prediction method. This is a fundamental difference with other state-of-the-art autoscalers that use bid prediction methods [3, 11].

On the other hand, it is also important to remark that devising a full-ahead scaling plan for the entire workflow execution is impracticable since it might lead to serious losses on makespan and/or cost. The reasons are, first, because cloud's performance is variable and therefore the exact duration of tasks is unpredictable, and second, because the occurrence of OOB errors is also unpredictable: given bid the occurrence of $\mathrm{OOB}$ errors depends on the future - and unknown- progression of spot prices.

For the above-mentioned reasons, CMI operates in an online way by alternating the search of scaling plans on one hand and the scheduling of tasks on the other hand. In this manner, decisions taken are suited to the actual progression of the workflow execution and the infrastructure, which leads to better makespan and cost savings.

CMI, and most autoscalers, operate during the entire execution of the workflow by periodically solving various autoscaling subproblems along the time in order to obtain the adequate scaling plan and task scheduling according to workflow execution progress and cloud infrastructure load state. Then, in subsections 2.1 and 2.2 we present the mathematical formulation that defines each one of the autoscaling subproblems that need to be solved during the workflow's execution, and the CMI autoscaler, respectively.

\subsection{Mathematical Formulation of Subproblems}

As we discussed before, the autoscaling process adapts the infrastructure periodically by solving a series of autoscaling subproblems subject to the current infrastructure state and the execution progress of the workflow. Each subproblem consist on determining a scaling plan that specifies the amount of ondemand and spot instances of each type, as well as the bids for such spot instances. Given $I$, the set of instance types considered for autoscaling, and $n=|I|$ the amount of instance types, we define a scaling plan $X$ is formally defined as a 3 -tuple $\left(\boldsymbol{x}^{\text {od }}, \boldsymbol{x}^{s}, \boldsymbol{x}^{b}\right)$, where:

- $\boldsymbol{x}^{\text {od }}=\left(x_{1}^{\text {od }}, x_{2}^{\text {od }}, \ldots, x_{n}^{\text {od }}\right) \in \mathbb{N}_{0}^{n}$ is a vector that describes the amount of on-demand instances for each of the $n$ instance types, each component ranges between 0 and the maximum number of instances that establishes the cloud provider,

- $\boldsymbol{x}^{s}=\left(x_{1}^{s}, x_{2}^{s}, \ldots, x_{n}^{s}\right) \in \mathbb{N}_{0}^{n}$ is a vector that describes the amount of spot instances for each of the $n$ instance types, each component ranges between 0 and the maximum number of instances that establishes the cloud provider, and

- $\boldsymbol{x}^{b}=\left(x_{1}^{b}, x_{2}^{b}, \ldots, x_{n}^{b}\right) \in \mathbb{R}_{\geq 0}^{n}$ is a vector of real values greater or equal to zero that represents the bid price for the the spot instances described on $\boldsymbol{x}^{s}$, each value $x_{i}^{b}$ is the bid for the $x_{i}^{s}$ instances of type $i$, each component ranges between the actual spot price and the on-demand price for such instance type.

Moreover, given the set of workflow tasks considered in the current autoscaling step, $\tau^{\text {period }}$, and the set of available instance types, $I$, a multi-objective autoscaling subproblem is defined as the minimization of the following three functions showed in Eq. (1):

$$
\min (\operatorname{makespan}(X), \operatorname{cost}(X), \operatorname{errorsImpact}(X)),
$$

subject to a set of constraints that restrict the feasible solution space and define the current status of the execution at the time that the the subproblem is being solved. 
1) Makespan. The makespan(.) function from Eq. (2) represents the expected execution time of the period tasks $\tau^{\text {period. }}$. To compute a solution's makespan, the model simulates the behavior of the scheduling algorithm considering the tasks in $\tau^{\text {period }}$ and the instances described in $\boldsymbol{x}^{\text {od }}$ and $\boldsymbol{x}^{s}$. Tasks are scheduled starting by those with lower margin for delays without violating the precedence order dictated by the workflow dependencies. Then, for each task the earliest completion time (ECT) criterion is used to select the most appropriate instance. Note that this criterion permits the assignment of tasks to instances that are not the ones that offer the shortest execution times but those which promise the earliest completion times.

Formally, a solution's makespan is computed as:

$$
\operatorname{makespan}(X)=\max _{t \in \tau^{\text {period }}}\left\{\operatorname{EST}(t)+d_{t}\right\}-\min _{t \in \tau^{\text {period }}}\{\operatorname{EST}(t)\}
$$

where $d_{t}$ is the duration of a task $t$ which in practice is mostly unknown and hence should be estimated by using a performance prediction mechanism [12]. Here, durations are estimated considering the ECT processor from the instances described in $\boldsymbol{x}^{o d}$ and $\boldsymbol{x}^{s}$. EST is the earliest start time and accounts for the minimum time at which a task can start its execution taking into account its parent tasks, i.e. the preceding tasks according to workflow dependencies. Moreover, the EST of a waiting task $t$ is computed as $\operatorname{EST}(t)=\max _{1 \leq k \leq p}\left\{\operatorname{EST}\left(t_{k}\right)+d_{k}\right\}$, where $t$ is a waiting task, $t_{k}$ is one of the $p$ parent tasks of $t$ and $d_{k}$ is the estimated duration of $t_{k}$. Then, for tasks that are ready to execute, the EST is set to the current time, i.e. when the current autoscaling subproblem is being solved.

Please note that the makespan function represents a very rugged optimization hyper-surface because it heavily depends on (a) the heterogeneity of tasks as the different possible assignments of tasks-instances provide a wide spectrum of durations, and (b) the workflow dependencies as they determine different patterns of tasks sequence/parallelism and propagate delays from parent to children tasks in cascade. Both of these characteristics, tasks heterogeneity and dependencies between them, make workflow autoscaling a particularly challenging problem. This is an important difference with respect to our previous work in [4] where we had focused on applications that comprise a set of homogeneous and independent tasks (without dependences among them), which determined a simpler autoscaling scenario.

2) Cost. The $\operatorname{cost}(\cdot)$ function from Eq. (3) represents the cost of running all the instances for one hour of computation.

$$
\operatorname{cost}(X)=\sum_{i=1}^{n} x_{i}^{o d} \cdot \operatorname{price}_{i}+x_{i}^{s} \cdot x_{i}^{b}
$$

In the case of on-demand instances, the model computes the total cost of executing all the instances of type $i\left(x_{i}^{o d}\right)$ multiplied by their corresponding on-demand price (price ${ }_{i}$ ). In the case of spot instances, the model estimates the cost in a different way. As the price of spot instances is determined by the cloud provider in a unpredictable way, the model estimates the cost by multiplying the amount of instances of type $i\left(x_{i}^{s}\right)$ by their corresponding bid $\left(x_{i}^{b}\right)$. In this estimation we also assume that OOB errors do not occur.

Note these two assumptions regarding the cost of spot instances lead in our approach to a pessimistic estimation of the overall cost because of the following two reasons. First, as OOB errors are not considered (although they could indeed occur during the actual execution) the cost of the hypothetically failing instances will be added up to the overall cost. Second, for each instance we used the bid as the spot price which is an upper bound of the actual spot prices.

3) Potential impact of $O O B$ errors. The errorsImpact $(\cdot)$ function from Eq. (4) estimates the potential impact on the workflow considering the amount of spot instances and their associated bids. As the occurrence of OOB errors depends on the chosen bid and the future evolution of spot prices, it cannot be exactly determined when and for which bids, OOB errors will occur. For such reason, and to cope with this source of unavoidable uncertainty, our formulation uses a probabilistic function of OOB-error occurrences. Formally, the OOB errors are computed as:

$$
\operatorname{errorsImpact}(X)=\sum_{i=1}^{n} x_{i}^{s} \cdot \operatorname{vCPU}_{i} \cdot P_{i}\left(x_{i}^{b}\right),
$$


To estimate the potential impact of OOB errors derived from choosing a given bid $\left(x_{i}^{b}\right)$ for the amount of instances of type $i\left(x_{i}^{s}\right)$, the errorsImpact $(\cdot)$ function computes a weighted sum of the OOB error probabilities $P_{i}\left(x_{i}^{b}\right)$. The probability of OOB errors for each instance type $i$ given the selected bid, $P_{i}\left(x_{i}^{b}\right)$, is multiplied by the maximum number of tasks that could be executing on the instances of such type. As a processor of any instance can not execute more than one task at a time, the maximum number of tasks that might be executed on the instances of the $i^{\text {th }}$ type is simply the total amount of processors of the spot instances such type, formally, $x_{i}^{s} \cdot \mathrm{vCPU}{ }_{i}$. In this way the function estimates the potential impact related to the number of tasks which can fail due to an OOB error. The objective function computes the overall impact of $\mathrm{OOB}$ errors considering all the instance types.

Constraints. The mathematical formulation for each subproblem also includes the following constraints:

$$
\begin{gathered}
\operatorname{cost}(X) \leq \mathrm{B}, \\
\mathrm{X}_{i}^{\text {min }} \leq x_{i}^{\text {od }}+x_{i}^{\mathrm{s}} \leq \mathrm{X}_{i}^{\max }, \\
\sum_{i=1}^{n} x_{i}^{o d}+x_{i}^{s}>0, \\
\mathrm{~S}_{\mathrm{i}}^{\text {currentPrice }} \leq \mathrm{x}_{\mathrm{i}}^{b} \leq \text { price }_{\mathrm{i}},
\end{gathered}
$$

where $\mathrm{X}_{i}^{\min }$ and $\mathrm{X}_{i}^{\max }$ are, respectively, the minimum and maximum number of allowed instances for the $i^{\text {th }}$ instance type, $\mathrm{S}_{i}^{\text {currentPrice }}$ is the current price for the spot instances of type $i$, price ${ }_{i}$ is the ondemand price for the $i^{\text {th }}$ instance type. The minimum amount of instances $\left(\mathrm{X}_{i}^{\mathrm{min}}\right)$ is the amount of running instances that are processing at least one task for each instance type as we can not terminate instances that are executing tasks.

The budget constraint in Eq. (5) limits the cost of the current autoscaling solution to be below the maximum monetary budget $B$. The $n$ constraints represented by Eq. (6) determine the minimum and maximum number of instances for each type. The constraint in Eq. (7) forces that autoscaling solutions must consider at least one instance. Finally, the $n$ constraints in Eq. (8) circumscribe bids to be between the current spot price, $\mathrm{S}_{i}^{\text {currentPrice }}$, and a maximum spot price, $\mathrm{S}_{i}^{\max }$, for each instance type.

\subsection{Autoscaler}

During the execution of a given workflow, the CMI autoscaler iteratively solves different cases of the multi-objective subproblems, which are defined by the current state of the workflow execution (i.e. the instances running, the degree of advance of tasks, and the current bid prices). In order to solve each autoscaling subproblem, CMI exploits the well-known multi-objective evolutionary algorithm called Nondominated Sorting Genetic Algorithm II (NSGA-II) [13]. CMI finds a set of Pareto-optimal solutions and then chooses the one that best fulfills a pre-established selection criterion. Then, CMI applies the scaling plan corresponding to such solution and schedules the tasks accordingly. Figure 1 1 illustrates the complete autoscaling process carried out by CMI.

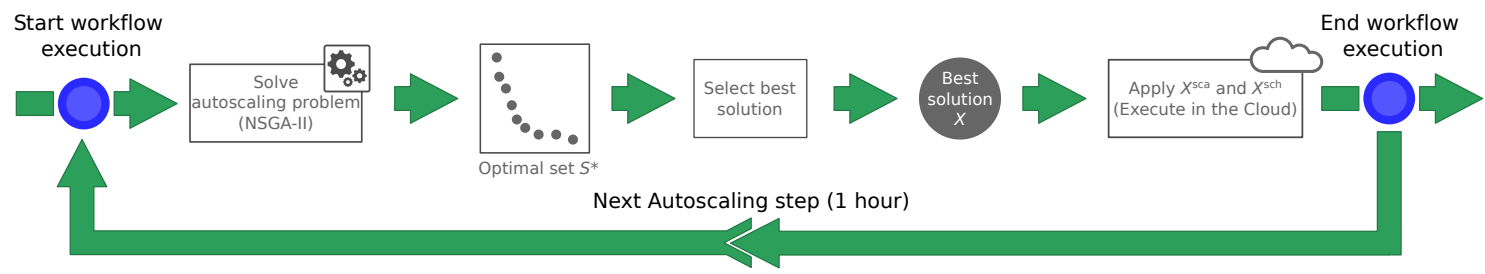

Figure 1: Illustration of the complete autoscaling process of CMI.

From the figure it can be seen that at each autoscaling step, CMI goes through three phases:

1. solving the multi-objective autoscaling subproblem at hand using the NSGA-II algorithm to obtain a set of Pareto-optimal solutions $\mathcal{S}^{*}$,

2. selecting a solution $\boldsymbol{x}$ according to a user-defined criterion, and

3. constructing and applying the resulting scaling plan $X$ and schedules tasks. 


\subsubsection{Solving the Multi-objective Subproblem}

NSGA-II [13] is a popular multi-objective evolutionary algorithm that has been applied to many real world problems [14, 15, 16]. NSGA-II initializes the population randomly according to the variable ranges for the given autoscaling subproblem. Then, the population is sorted based on non-domination in a series of fronts. The first front is a completely non-dominant set for the current population and the second front is dominated by the individuals only in the first front, and so on. The fitness of each individual corresponds to number of front it belongs to. Crowding distance is also calculated for each individual. Such distance measures the distance between an individual and its neighbors. Parents are selected from the population by using binary tournament based on both, fitness and crowding distance, which promotes the selection of nondominated diverse solutions. The selected parents produce an offspring by applying the Simulated Binary Crossover (SBX) operator, which attempts to simulate the offspring distribution of binary-encoded singlepoint crossover on real-valued variables. Resulting individuals are also mutated by applying the Polynomial Mutation (PM) operator, which attempts to simulate the offspring distribution of binary-encoded bit-flip mutation on real-valued variables. These two operators favor offspring nearer to the parent.

Individuals in the current population and current offsprings are sorted based on non-domination and only the best $k$ individuals are selected based on fitness and crowding distance as indicated earlier. In this context, $k$ is the population size. Finally, the algorithm terminates its execution once the maximum number of evaluations for the generated individuals is met, returning the non-dominated solutions obtained so far.

\subsubsection{Selection of the Best Solution}

From the solutions in the optimal set $\mathcal{S}^{*}$ obtained by NSGA-II, CMI applies a global criterion method to select one of the candidate solutions. This strategy permits obtaining a solution in an autonomous way without relying on an human decision maker. The adopted selection criterion is the following: $\arg \min _{\boldsymbol{x}}\left\|f(\boldsymbol{x})-\boldsymbol{z}^{\text {ideal }}\right\|$, where $\boldsymbol{x} \in \mathcal{S}^{*}$. This method selects the solution $\boldsymbol{x}$ that minimizes the distance to an ideal solution $\boldsymbol{z}^{\text {ideal }}$, where $\|\cdot\|$ is the $L_{2}$ norm. We choose $\boldsymbol{z}^{\text {ideal }}=(0,0,0)$ as the ideal solution, which corresponds to the solution with failure probability, makespan and cost equal to 0 . This policy tends to select the most balanced solution considering the values for each one of the objectives.

\subsubsection{Scaling Plan and Task Scheduling}

Based on the selected solution $x$, the autoscaler reconstructs the scaling plan. The autoscaler acquires the amount of on-demand $\left(\boldsymbol{x}^{\text {od }}\right)$ and spot instances $\left(\boldsymbol{x}^{s}\right)$ with the corresponding bid prices $\left(\boldsymbol{x}^{b}\right)$ indicated in the selected solution $\boldsymbol{x}$. Then, tasks in $\tau^{\text {period }}$ are scheduled using the ECT policy on the requested instances acquired according to the scaling plan.

For validating the described autoscaler we performed a comparative study against a state-of-the-art autoscaler called SIAA [3] over four real world workflow applications from the Astronomy and Environmental Engineering domains. Next section describes the main characteristics of these applications.

\section{Study Cases}

This Section describes four scientific workflows that serve as different study cases due to their own particular characteristics, mainly in terms of: $(i)$ the ranges of task durations -from a few minutes of computation in some workflows to days in others-, and (ii) the structure of workflow dependencies, which determine very different patterns and possibilities of task execution parallelism.

These workflows come from the areas of Astronomy and Environmental Engineering [9, 1] and were used to validate the performance of the autoscaling strategies under realistic workload patterns. The scientific workflows are described below:

CyberShake CyberShake is a workflow used in the Southern California Earthquake Center (SCEC) ${ }^{4}$ for characterizing hazards generated by earthquakes. CyberShake performs a Probabilistic Seismic Hazard Analysis (PSHA) over a geographic region. Message Passing Interface (MPI) tasks performing finite difference simulations are executed to generate Strain Green Tensors (SGTs). The generated

\footnotetext{
${ }^{4}$ Southern California Earthquake Center: http: //www.scec.org
} 
SGT data is used to compute synthetic seismograms for each of the predicted ruptures. Finally, spectral acceleration and probabilistic hazard curves are generated.

LIGO's Inspiral Laser Interferometer Gravitational Wave Observatory (LIGO) 5 is in the crusade for the detection of gravitational waves resulting from several events in the universe according to Einstein's theory of general relativity. The LIGO Inspiral Analysis Workflow (LIGO's Inspiral) analyzes data from the coalescing of binary neutron stars and black holes. Time-frequency data from events captured by each of the three LIGO detectors is split into smaller chunks for analysis. Each chunk is used to generate a subset of waveforms within a given parameter space later used for computing a matched filter output. When a true inspiral is detected a trigger is generated and it is checked against triggers from the other detectors. From now on we will refer to this workflow simply as Inspiral.

Montage Montage is a toolkit created by the Infrared Processing and Analysis Center (IPAC) ${ }^{6}$ of the NASA 7 aimed to generate custom mosaics of the sky from a set of input images. The geometry of such input images is used to compute the geometry of the final mosaic. The geometry is used to reproject the images into the same scale and rotation. Then, the images are corrected for standardizing the different background emissions to the same level. Re-projected and corrected images are added together into the final mosaic.

Pan-STARRS Panoramic Survey Telescope And Rapid Response System (Pan-STARRS's) project ${ }^{8}$ is a continuous survey of the entire sky. The data are collected by a telescope and used to detect hazardous objects in the Solar System, and other astronomical studies including cosmology and Solar System astronomy. The astronomy data from Pan-STARRS is managed through PSLoad and PSMerge workflows. The PSLoad stages incoming data files from the telescope pipeline and loads them into individual relational databases each night. Periodically the online production databases that can be queried by practitioners, are updated with the databases collected over the week by a second workflow called PSMerge. Both PSLoad and PSMerge workflows are data intensive but require coordination and orchestration of resources to ensure reliability and integrity of the data products.

These workflows present differences on their structure and alternate phases of parallel and sequential tasks, which in principle require a timely and precise scaling up or down of the cloud infrastructure to properly reduce makespan and cost. An additional aspect to take into consideration in this analysis is the duration of tasks that, in conjunction with the mentioned structural characteristics of the workflow, will determine the preference over parallel/sequential execution of tasks, predominance of on-demand versus spot instances, and selection of higher or lower bid prices to request spot instances. All these factors shape a complex spectrum of execution conditions that an autoscaler must consider to properly conduct the execution.

Note that these different workload and parallelism patterns condition the possibilities of instances acquisition and tasks scheduling while trying to minimize makespan, cost and number of OOB errors. For example multiple parallel short-duration tasks are best suitable for spot instances at low bids as the potential of cost and makespan reduction is large and subject to a low instance failure probability. Conversely, long-duration critical tasks are best suitable for on-demand instances, which are more reliable (as they are not subject to OOB errors) but are more expensive.

\section{NSGA-II Parameterization}

In order to obtain the best NSGA-II parameterization for each studied workflow we performed a sensitivity analysis by sampling 140 parameter configurations using the Saltelli sampling method [17] and performing 30 repetitions for each configuration using different random number generator seeds. The following list presents the NSGA-II parameter exploration ranges:

\footnotetext{
${ }^{5}$ LIGO's Inspiral: http://www.ligo.caltech.edu/

${ }^{6}$ IPAC: http:// www.ipac.caltech.edu/

${ }^{7}$ NASA: http://www. nasa.gov/

${ }^{8}$ Pan-STARRS: http://www.ifa.hawaii.edu/
} 
- maxEvaluations (max eval.): the number of solutions to be evaluated before terminating the algorithm. Sampling interval: [500, 10000], step 1.

- populationSize (pop. size): the number of solutions to keep across generations. Sampling interval: [100, 400], step 1.

- sbx.rate (SBX rate): the probability of applying the Simulated Binary Crossover (SBX) on a pair of individuals. Sampling interval: [0.8, 0.9], step 0.01.

- sbx.distributionIndex (SBX dist.): The distribution index controls the shape of the offspring distribution. Larger values for the distribution index generates offspring closer to the parents. Sampling interval: $[5.0,20.0]$, step 0.01 .

- pm.rate (PM rate): the probability of applying the Polynomial Mutation (PM) on each individual. Sampling interval: $[0.5,0.6]$, step 0.01 .

- pm.distributionIndex (PM dist.): As in the case of SBX, the distribution index controls the shape of the offspring distribution. Larger values for the distribution index generates offspring closer to the parents. Sampling interval: $[10.0,15.0]$, step 0.01 .

Figure 2 presents the combined Pareto fronts resulting from the samplings and seeds used for each workflow. For the sake of clarity, scatter pots exclude extreme solutions, i.e. those solutions with extreme high cost or high makespan. Note that we can allow us this simplification as the aim of our autoscaler is to produce balanced solutions regarding the modeled problem objectives. As the solution method selector described in Section 2.2.2 chooses solutions closer to $(0,0,0)$, we can focus on the portion of the Pareto-fronts closer to the origin.

(a) CyberShake

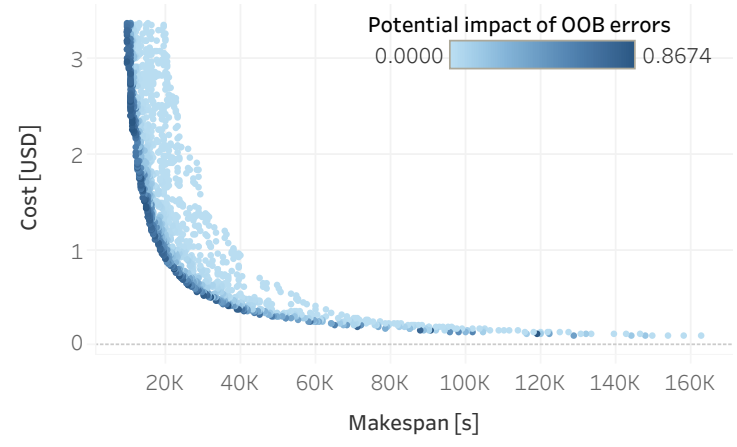

(c) Montage

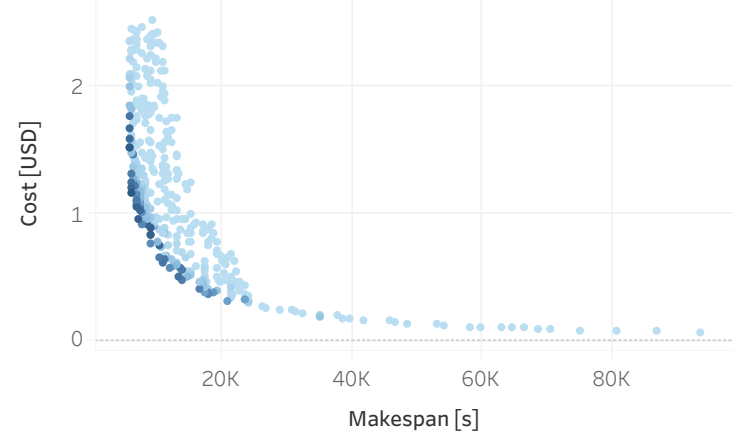

(b) Inspiral

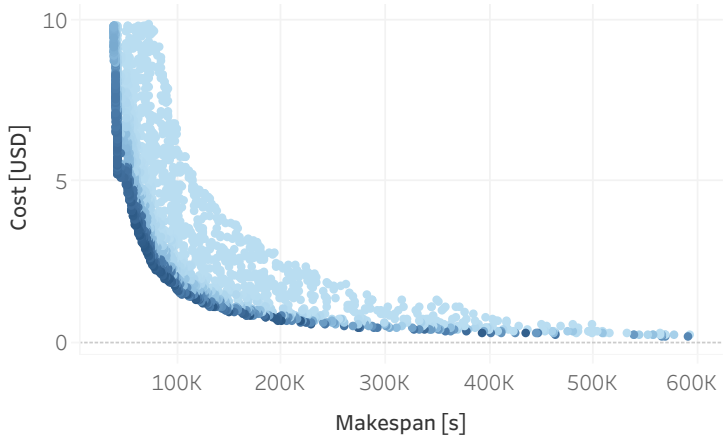

(d) Pan-STARRS

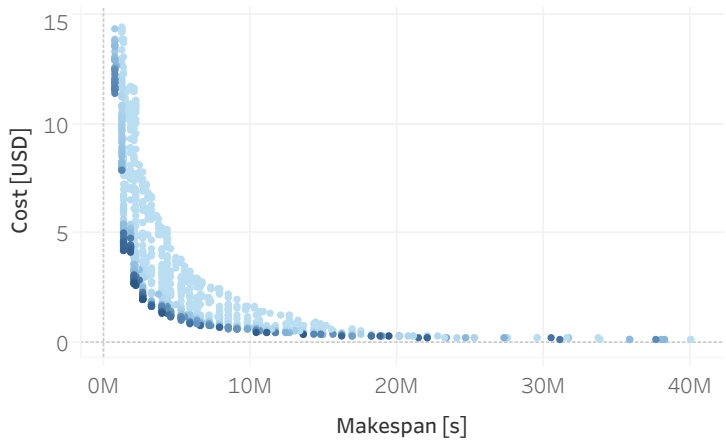

Figure 2: Combined 3D Pareto fronts for each workflow obtained by NSGA-II. Fronts were obtained by the combination of solutions obtained for all the parameter samples (140) and seeds (30). Potential impact of OOB errors are presented by the intensity of color. 
From all the evaluated parameter samples and repetitions we applied the Sobol method for variancebased sensitivity analysis [18]. In general terms, this method decomposes the variance of a model's output into portions associated to the sets of inputs for such model. In the context of this study, we are interested in analyzing the effect of the NSGA-II parameters on the variance of the NSGA-II performance. Therefore we choose the hypervolume (HV) metric of the obtained Pareto-fronts as the performance indicator, as it captures the degree to which obtained solutions are close to a reference set and how they are distributed.

The Sobol method is useful because it permits measuring the sensitivity of NSGA-II across the space of parameters discriminating the effects of different subsets of parameters. In this study we focus on the analysis of total-order effects, which measure the contribution of each parameter to the output variance, which includes the variance caused by any of its interactions of any order with any other parameter. In this context, order indicates the number of variables considered in the interactions, first-order refers to the effects of a single variable, second order refers to the effects of pairs of variables and so on. Table 1 presents the results of the sensitivity analysis for each workflow.

Table 1: Sensitivity analysis results for NSGA-II discriminated by workflow. Values represent the total-order effects of each of the parameters NSGA-II parameters: maxEvaluations (max eval.), populationSize (pop. size), sbx.rate (SBX rate), sbx.distributionIndex (SBX dist.), pm.distributionIndex (PM dist.), pm.rate (PM rate) and pm.distributionIndex (PM dist.). Parameters max eval. and pop. size are the most sensitive ones for every studied workflow.

\begin{tabular}{|c|c|c|c|c|c|c|}
\hline \multirow{2}{*}{ Workflow } & \multirow{2}{*}{ max eval. } & \multirow{2}{*}{ pop. size } & \multicolumn{2}{|c|}{ SBX } & \multicolumn{2}{|c|}{ PM } \\
\hline & & & rate & dist. & rate & dist. \\
\hline CyberShake & 1.141 & 0.123 & -0.025 & -0.008 & 0.006 & -0.044 \\
\hline Inspiral & 1.104 & 0.099 & -0.033 & 0.020 & 0.033 & 0.091 \\
\hline Montage & 1.187 & 0.151 & 0.019 & 0.064 & 0.099 & 0.124 \\
\hline Pan-STARRS & 1.139 & 0.129 & -0.027 & -0.042 & -0.050 & -0.123 \\
\hline
\end{tabular}

From the table it can be seen that max eval. and pop. size are the most sensitive parameters (indicated by the large values). These results indicate that the different values that such parameters might take have strong effect on the resulting HV values, i.e. on the performance of NSGA-II. In other words, selecting inappropriate values for such parameters will inhibit NSGA-II from obtaining good quality solution sets.

The lower sensitivity values taken by the remaining parameters indicate that in terms of relevance their effects are smaller than the effects of the first cited parameters. Nonetheless, for the proper operation of NSGA-II, it is also important to determine the right values of such parameters.

With the aim of obtaining the best parameterization of NSGA-II for the autoscaler we choose the one that maximizes the HV metric. By maximizing the HV we ensure that the parameters set selected promise a good level of convergence and diversity on the solutions with respect to the reference sets. Table 2 reports the selected NSGA-II parameterization and the corresponding HV value for each workflow.

Table 2: Selected parameters for each workflow.

\begin{tabular}{|c|c|c|c|c|c|c|c|}
\hline \multirow{2}{*}{ Workflow } & \multirow{2}{*}{ max eval. } & \multirow{2}{*}{ pop. size } & \multicolumn{2}{|c|}{ SBX } & \multicolumn{2}{|c|}{$\mathrm{PM}$} & \multirow{2}{*}{ HV } \\
\hline & & & rate & dist. & rate & dist. & \\
\hline CyberShake & 9712 & 145 & 0.88 & 18.9 & 0.55 & 10.8 & 0.994 \\
\hline Inspiral & 9712 & 145 & 0.82 & 17.2 & 0.58 & 12.5 & 0.997 \\
\hline Montage & 9712 & 145 & 0.88 & 18.9 & 0.55 & 10.8 & 0.996 \\
\hline Pan-STARRS & 9712 & 145 & 0.88 & 18.9 & 0.55 & 10.8 & 0.990 \\
\hline
\end{tabular}

Note that for the most sensitive parameters, max eval. and pop. size, the selected configurations include those values closer to the upper bound of the parameterization ranges presented at the beginning of this section. These values make the genetic algorithm to perform the search working on a larger pool of solutions and letting those solutions evolve through a larger number of generations, which leads to the obtainment of better sets of candidate solutions for the autoscaling problems. These parameters are used in the comparative study presented in the next section. 


\section{Comparative study}

In order to evaluate the performance of CMI we compare it with another state-of-the-art autoscaler named Spot Instances Aware Autoscaling (SIAA), which is described in subsection 5.1 Then, details on the experimental settings and experimental results are provided in subsections 5.2 and 5.3, respectively. Finally, an analysis of statistical significance of results is provided in subsection 5.4

\subsection{Spot Instances Aware Autoscaling (SIAA)}

SIAA [3] autoscaler starts determining a scaling plan and then schedules the tasks for running on the available instances. To this end, SIAA uses, on the one hand, a scaling algorithm which permits the acquisition of a cloud infrastructure comprising on-demand and spot instances, and on the other hand, an heuristic scheduling algorithm to minimize the workflow makespan and reduce the probability that failing spot instances might interrupt the execution of longer tasks. In the scaling phase, SIAA determines a consumption vector and then scales it to meet the budget constraint. To such end, the available budget is split in two parts and assigned to acquire the maximum amount of on-demand and spot instances according to such budget distribution. To do this, SIAA uses bidding methods to request spot instances.

In this work SIAA uses different configurations of the probabilistic bidding method discussed on [3], which aim at bidding prices that promise bid failures below a given probability threshold that we call confidence level. Then, in the scheduling phase, the algorithm tackles the minimization of the workflow makespan by reducing the execution time of critical tasks. Moreover, the slack times (the maximum amount of time that a task can be delayed without delaying any of their child tasks) are used to determines a priority for the selection of tasks during scheduling. The tasks which are ready to execute are sorted by their slack times in an increasing order (first those tasks which have smaller margin for delay). This criterion executes first those tasks which potentially have more impact on the workflow makespan.

The algorithm schedules the tasks -one by one- until there are no more ready tasks to schedule or all the instances are busy. Tasks are scheduled to instances according to two criteria. First, tasks are allocated to on-demand instances, but if there are no available instances, the algorithm checks the availability of spot instances. This policy favors that failures, if occur, affect non-critical tasks that could be re-scheduled without a large impact on the overall makespan as they have a wider margin for delays (larger slack times). Second, tasks are allocated to the instances that promise the ECT. This policy is applied while selecting an on-demand or a spot instance for the task at hand. In this way the algorithm promotes the execution of tasks on the fastest possible instances.

\subsection{Experimental Settings}

In order to perform the experiments we instantiated the CloudSim toolkit [19], which is heavily used within the community for the design of realistic cloud experiments. The characteristics and the on-demand price of the instances considered during the experimentation are presented in Table 3 along with their corresponding on-demand price. Values in the table correspond to real characteristics of Amazon Elastic Compute Cloud (EC2) instances, where vCPU is the number of (virtual) CPUs available for each one of the instance types, $\mathrm{ECU}^{\mathrm{tot}}$ (acronym for $E \mathrm{C} 2$ Compute $U$ nits) is the relative computing power of the instances considering all its virtual CPU $\$$ ECU is the relative performance of each one of the CPUs, and the last column shows the price in United States dollars (USD) per hour of computation. These instance types were selected to provide diverse spectrum of performance and price configurations.

For experimentation we used an actual history of Amazon EC2 spot prices that corresponds to the period between March $7^{\text {th }}$ and June $7^{\text {th }}$ of 2016 for the US-west region (Oregon) [20].

The first two months of data were used to compute the OOB errors probabilities by counting the number of times a sliding time window of 1 hour presented spot prices over the set of bid values. Note that these bid values correspond to the bid levels defined in our mathematical formulation of the scheduling problem. The data corresponding to the last month of the history was kept for the experiments presented on Section 5 as the spot price variations during the simulation. Using the data in such way allowed us to perform

${ }^{9}$ One ECU is equivalent to a CPU capacity of a 1.0-1.2 GHz 2007 Opteron 
Table 3: Characteristics of the on-demand instances. The data corresponds to instances belonging to the US-west (Oregon) region.

\begin{tabular}{lcccc}
\hline Instance type & vCPU & ECU $^{\text {tot }}$ & ECU & Price (USD) \\
\hline \hline t2.micro & 1 & 1 & 1 & $\$ 0.013$ \\
m3.medium & 1 & 3 & 2 & $\$ 0.07$ \\
c3.2xlarge & 8 & 28 & 3.5 & $\$ 0.42$ \\
r3.xlarge & 4 & 13 & 3.25 & $\$ 0.35$ \\
m3.2xlarge & 8 & 26 & 3.25 & $\$ 0.56$ \\
\hline
\end{tabular}

the evaluation of the bidding methods and therefore the performance of the autoscaling strategies while completely ignoring future evolution of spot prices, which is the real scenario for any EC2 user.

We configured our competitor, SIAA, by varying the proportion of spots and on-demand instances, i.e. spots ratio (SR) and the bidding method confidence (BMC), which is the bid price that probability of OOB errors the same bidding method used in [3]. The values that $\mathrm{SR}$ can take are $0.1,0.2, \ldots, 1.0$ and the values taken by BMC are $0.05,0.1, \ldots, 0.25$, which gives a total of 55 different configurations of SIAA (11 SR values $\times 5 \mathrm{BMC}$ values). To ensure the robustness of results we repeated (30 times) the simulations of CMI, and SIAA for each of the 55 configurations, giving a total of 30 executions of CMI and 1650 of SIAA for each workflow. These configurations were used for each of the 4 studied workflows giving a total of $30 \times 4+1650 \times 4=6720$ simulations.

\subsection{Experimental Results}

Table 4 presents the mean values for the number of on-demand and spot instances, makespan in seconds, cost in USD, number of task failures, and the $L_{2}$-norm considering the task failures, makespan and cost resulting from the simulation, which provides a joint analysis of the metrics of interest for this autoscaling problem.

Table 4: Summary of results. Each row presents the mean values per strategy considering all the studied workflows. Except for the average number of on-demand and spot instances that are merely descriptive, for the remaining metrics lower values represent better results.

\begin{tabular}{lccccccc}
\hline Workflow & Strategy & $\begin{array}{c}\text { No. of } \\
\text { on-demand }\end{array}$ & $\begin{array}{c}\text { No. of } \\
\text { spot }\end{array}$ & Makespan & Cost & $\begin{array}{c}\text { Task } \\
\text { Failures }\end{array}$ & $L_{2}$ norm \\
\hline \hline CyberShake & CMI & 6.37 & 85.7 & 18604.02 & $\mathbf{8 . 4 0}$ & 47.33 & $\mathbf{0 . 2 2}$ \\
& SIAA & 7.08 & 22.13 & $\mathbf{1 8 4 1 9 . 2 1}$ & 13.62 & 33.13 & 0.71 \\
\hline Inspiral & CMI & 10.59 & 133.26 & $\mathbf{1 0 8 9 5 8 . 5 6}$ & $\mathbf{6 5 . 3 8}$ & 110.41 & $\mathbf{0 . 1 4}$ \\
& SIAA & 24.26 & 51.88 & 109494.01 & 155.21 & 135.75 & 0.72 \\
\hline \multirow{2}{*}{ Montage } & CMI & 4.72 & 95.62 & $\mathbf{2 9 9 6 2 . 6 8}$ & $\mathbf{4 . 4 7}$ & 74.66 & $\mathbf{0 . 1 0}$ \\
& SIAA & 6.89 & 13.37 & 35806.54 & 8.51 & 30.52 & 0.80 \\
\hline Pan-STARRS & CMI & 73.52 & 712.12 & 2215656.73 & $\mathbf{3 3 5 1 . 6 1}$ & 1579.68 & $\mathbf{0 . 3 9}$ \\
& SIAA & 49.06 & 105.32 & $\mathbf{1 7 3 3 4 3 1 . 7 2}$ & 6206.71 & 397.23 & 0.63 \\
\hline
\end{tabular}

From the table it can be seen that CMI tends to use a larger number of spot instances than SIAA. Considering makespan, and in the case of CyberShake and Montage, CMI obtains better average performance than SIAA. The opposite is true for the remaining workflows. In terms of cost, CMI outperforms SIAA for all the workflows and with a wide margin of savings (around 50\% in some cases). Note that the number of task failures is also much higher for CMI than for SIAA, which is explained by the larger number of spot instances used and the low bid prices used. The negative impact of such high number of task failures is evident for Inspiral and Pan-STARRS, which are the workflows with many very large tasks. However, a very interesting observation for the remaining workflows is that despite the high number of failures, makespan is shorter for CMI than for SIAA. The reason is CyberShake and Montage have many short-duration tasks, 
and if failures occur they do not heavily affect many tasks, even more, tasks completed in the last hour of execution of a failing spot instance are executed for free according to Amazon spot policies.

Finally the $L_{2}$-norm metric favors CMI in all the cases, which indicates that such autoscaler outperforms SIAA always in terms of cost and in two of the studied workflows in terms of makespan. For the remainder of this section we focus on the $L_{2}$-norm metric, which provides a joint measure of performance facilitating the comparison procedure. Analyzing makespan or cost in isolation does not capture the trade-off that occurs between those metrics, thus limiting the depth of the derived conclusions.

We can conclude that CMI is not restricted by any proportion of spot and on-demand instances nor by the bid prices to use, which permits acquiring different distributions of instances depending on the next workload pattern. In addition, CMI also determines the bid price to use for each instance type, which permits adjusting the relation between the maximum price to pay and reliability according to the upcoming tasks.

\section{Analysis of the Autoscaling Process}

In this section we provide an analysis of the events that occur during the autoscaling process on a particular simulation scenario (i.e. same workflow and repetition number). We analyzed four autoscaling processes for the Montage workflow, one of them corresponding to CMI, and the others to SIAA with three different configurations. The first case corresponds to the process carried out by the best-performing CMI (CMI-b). Then, the remaining cases correspond to three of the 55 configurations of SIAA with: its best performing configuration (SIAA-b), a configuration that produced an expensive execution (SIAA-e), and with a configuration (SIAA-u) for which the execution is strongly affected by the unreliability of spot instances. Table 5 summarizes the performance metrics considering number of instances, virtual CPUs (vCPUs), makespan, cost, number of task failures and the $L_{2}$-norm metric for the analyzed cases.

Table 5: Metrics for the analyzed cases.

\begin{tabular}{lcccccc}
\hline Autoscaler & Instances & vCPUs & Makespan & Cost & OOB errors & $L_{2}$-norm \\
\hline \hline CMI-b & 301 & 450 & $\mathbf{2 9 2 6 8 . 3 3}$ & $\mathbf{3 . 2 1}$ & $\mathbf{0}$ & $\mathbf{0 . 0 4 5}$ \\
\hline SIAA-b & 26 & 168 & 29991.00 & 4.00 & $\mathbf{0}$ & 0.051 \\
SIAA-e & 26 & 168 & 29991.00 & 22.78 & $\mathbf{0}$ & 0.196 \\
SIAA-u & 27 & 176 & 38648.00 & 4.22 & 1 & 0.060 \\
\hline
\end{tabular}

In Figure 3 we present the autoscaling processes for the mentioned cases. Curves indicate the number of tasks and vCPUs through time, and labels indicate the accumulated cost of execution on each autoscaling step. From the figure it can be seen that:

1. $C M I-b$ achieves the best performance with respect to makespan and cost, and therefore also achieves the best performance with respect to the $L_{2}$-norm (see Table 5). Moreover, it can also be seen that CMI-b requests a greater number of VMs than the other three processes. However, this does not impact the cost because CMI-b chooses mostly spot instances and makes sure to take bids that do not produce OOB errors.

2. SIAA- $b$ (SIAA with the best performing configuration) presents a good balance between makespan and cost. As can be seen, SIAA-b is only outperformed by CMI-b. Concretely, the gains of CMI-b with respect to best-SIAA-b for the makespan and cost are equal to $2.41 \%$ and $19.75 \%$, respectively.

3. SIAA-e (SIAA with a configuration that produced an expensive execution) achieves the same makespan obtained by SIAA-b, however, it is almost 6 times more expensive than SIAA-b due to the fact that SIAA-e uses a large number of on-demand instances. Here, the gains of CMI-b with respect to SIAA-e are equal to $2.41 \%$ and $85.90 \%$ for the makespan and cost, respectively.

4. SIAA- $u$ (SIAA with a configuration for which the execution is strongly affected by the unreliability of spot instances) has the largest makespan in this analysis. In this case, an OOB error occurs producing the failure of a critical task, which delays their termination in detriment of workflow makespan. This event is illustrated with a vertical red line in the Figure 3. The obtained makespan is considerably greater than CMI-b and the other SIAA configurations. However, as can be seen in the Figure 3 the 


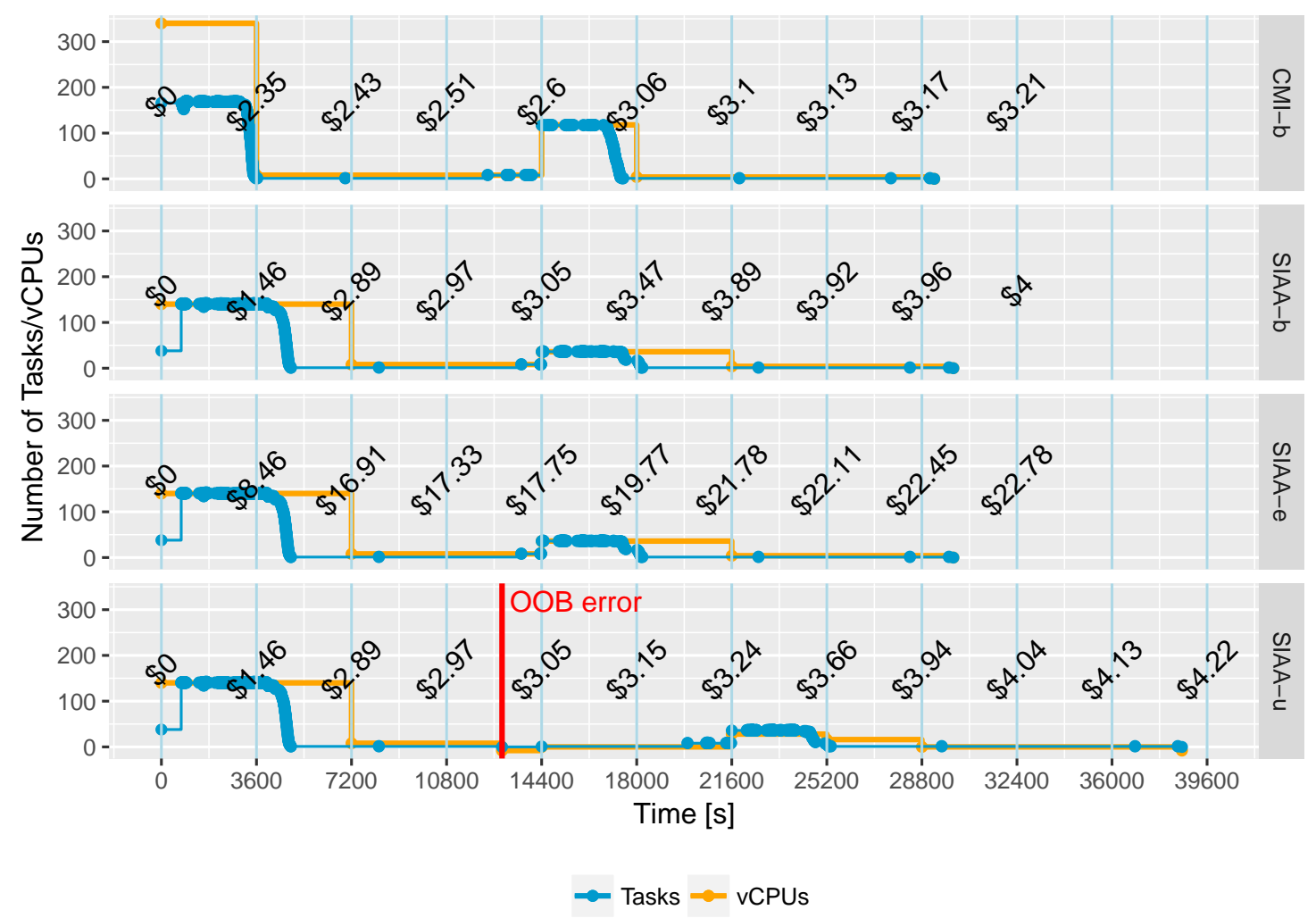

Figure 3: Analysis of the autoscaling process for Montage for CMI-b, the best performing configuration of SIAA (SIAA-b), a configuration of SIAA that produces a high cost of execution (SIAA-e), and a configuration of SIAA that produces an OOB error affecting the execution time (SIAA-u). The vertical red line indicates the occurrence of an OOB error during the execution of SIAA-u.

cost obtained by SIAA-u is quite close to SIAA-b. Finally, the gains obtained by CMI-b with respect to SIAA-u are equal to $24.27 \%$ and $23.93 \%$ with respect to the makespan and cost, respectively.

\subsection{Statistical Significance of Results}

In this subsection we show the statistical significance of the obtained results. Results are statistically significant when there is a possibility that a relationship between two or more variables is caused by something other than chance. The statistical hypothesis testing is used to determine if the result of a dataset is statistically significant. This test provides a p-value, representing the probability that a random chance could explain the result. This kind of analysis is fundamental to support the validity of the performance differences found between the analyzed autoscaling strategies.

Table 6 shows the mean, median, standard deviation (SD), min. and max. for the $L_{2}$-norm metric per workflow. Centrality metrics (mean and median) and max. values of the $L_{2}$-norm favors CMI in all cases, in terms of SD and min. value, CMI has the smallest values in most cases.

To evaluate the significance of the improvements achieved by CMI we applied the Mann-Whitney $U$ test [21], a non-parametric method that evaluates if two data samples come from the same population ${ }^{10}$ To ensure the validity of our claims we selected a strong confidence level of $\alpha=0.001$. We compared CMI versus each of the 55 configurations of SIAA. Table 7 summarizes the number of times CMI outperforms SIAA in terms of the $L_{2}$-norm, the number of times that improvement is significant and the range of percentual improvements for each application.

\footnotetext{
${ }^{10} \mathrm{As}$ the $L_{2}$-metric results are non-normally distributed (See Table 6 ) a non-parametric method is required to test the significance of the differences.
} 
Table 6: Detailed $L_{2}$-norm results. Each row presents the mean values per strategy considering all the studied applications. Best mean and median values are highlighted. Last column describes the result of the normality test Shapiro-Wilk with a confidence level of $\alpha=0.001$.

\begin{tabular}{lccccccc}
\hline Workflow & Strategy & Mean & Median & SD & Min. & Max. & Normal \\
\hline \hline CyberShake & CMI & $\mathbf{0 . 2 2}$ & $\mathbf{0 . 1 7}$ & 0.12 & 0.10 & 0.69 & no \\
& SIAA & 0.71 & 0.83 & 0.26 & 0.08 & 1.01 & no \\
\hline Inspiral & CMI & $\mathbf{0 . 1 4}$ & $\mathbf{0 . 0 9}$ & 0.16 & 0.01 & 0.64 & no \\
& SIAA & 0.72 & 0.85 & 0.25 & 0.02 & 1.15 & no \\
\hline Montage & CMI & $\mathbf{0 . 1 0}$ & $\mathbf{0 . 0 7}$ & 0.07 & 0.02 & 0.25 & no \\
& SIAA & 0.80 & 0.92 & 0.20 & 0.18 & 1.07 & no \\
\hline Pan-STARRS & CMI & $\mathbf{0 . 3 9}$ & $\mathbf{0 . 3 9}$ & 0.23 & 0.01 & 1.01 & yes \\
& SIAA & 0.63 & 0.66 & 0.14 & 0.27 & 1.03 & no \\
\hline
\end{tabular}

For each workflow, we carried out 11 tests corresponding to the comparison of CMI with each variant of SIAA for the different spot ratio (SR). In the table, the "Better" column describes the number of cases for which CMI presented significant improvements over the different SIAA configurations in terms of the $L_{2}$-norm. The "Non signif." column presents the number of cases in which there were not significant differences between CMI and SIAA in terms of the $L_{2}$-norm. The columns "Min impr." and "Max impr." show the range of percentual improvements that are significant according to the test. It is important to mention that in none of the cases SIAA presented statistically significant improvements over CMI.

Table 7: Summary of statistical test results (Mann-Whitney U test with a confidence level of $\alpha=0.001$ ). The amount of tests for which CMI present significant improvements over SIAA, are highlighted.

\begin{tabular}{lccccc}
\hline Workflow & Total no. of tests & Better & Non signif. & Min impr. & Max impr. \\
\hline \hline CyberShake & 11 & $\mathbf{1 0}$ & 1 & $14.09 \%$ & $70.24 \%$ \\
Inspiral & 11 & $\mathbf{1 1}$ & 0 & $31.54 \%$ & $80.04 \%$ \\
Montage & 11 & $\mathbf{1 1}$ & 0 & $34.68 \%$ & $85.77 \%$ \\
Pan-STARRS & 11 & $\mathbf{9}$ & 2 & $10.16 \%$ & $38.75 \%$ \\
\hline All & 44 & $\mathbf{4 1}$ & 3 & $10.16 \%$ & $85.77 \%$ \\
\hline
\end{tabular}

This is expected as the Pan-STARRS workflow comprises very long tasks (almost 7 days in the worst case), not captured by our definition of the probabilities computation since it considers one-hour time windows. Therefore our model greatly underestimates error probabilities for such tasks impacting mainly on makespan as we saw in Table 4 . We believe that simply computing probabilities in a more fine-grained way might allow us to obtain better autoscaling solutions allowing CMI to outperform SIAA with wider margins.

\section{Related Work}

In the literature there are many approaches addressing the efficient management of scientific applications on the cloud [22], however, to the best of our knowledge, there are no efforts covering autoscaling strategies based on an online multi-objective meta-heuristic for executing workflow applications, which additionally consider spot instances and minimize both the makespan, cost and probability of OOB errors. Among these works, we can mention our own past work in [3] where we proposed an autoscaler called SIAA for workflow applications which considered spot instances. The aim of [3] was to minimize the makespan subject to budget constraints (i.e. limiting the maximum cost). In such work, to cope with spot instances unreliability, tasks were heuristically scheduled with the aim of mitigating the negative effects of out-of-bid errors on the workflow makespan. The main difference with respect to the present paper is that in [3] the monetary cost was not considered. Then, in [8], the authors have proposed a cost-efficient based 
scheduling algorithm which allows to lease instances from clouds for executing scientific workflows while meeting the required deadlines of tasks. The tasks are scheduled according to the spot instance pricing. On the other hand, the work in [23] is focused on running large-scale computational applications on clouds, especially for on-demand instances and spot instances offered by Amazon EC2. In [23], after analyzing the characteristic of the spot price and the effect of spot instances disturbance, the authors proposed a dynamic approach for running the applications with the aim of reducing cost, increasing the reliability and reducing the complexity of fault tolerance without affecting the overall performance and scalability. However, the main difference between the works [8, 23] and ours is that we focus on a budget-constrained autoscaling problem while the efforts mentioned focus on solving scheduling problems subject to task deadline constraints, thus they are useful in different scenarios. Another important distinction is that we are also focused in reducing the probability of failures - to avoid heavily affecting workflow application results- and without relying on spot price predictors.

There are also some works particularly addressing workflow autoscaling [7, 24, 25, 10] with deadline or budget constraints. The problem was first addressed by [25] where the authors proposed an autoscaling strategy for the efficient execution of multiple workflow applications subject to deadline constraints. The goal of [25] was to ensure that all tasks finish before their respective deadlines by using the cheapest resources whenever possible. Later, the same authors moved to the problem of workflow autoscaling but considering budget constraints [10]. Another work is the one presented in [7], where the main characteristics of the tasks in the workflow structure are learned over time, i.e., the autoscaler dynamically adapts the number of allocated resources in order to meet the deadlines of all tasks without knowing the workflow structure itself and without any information of the execution time. The goal of this work was to minimize the makespan. These strategies were proposed for simultaneously addressing the problem of scaling down/up the virtual cloud infrastructure and scheduling workflow tasks in heterogeneous cloud infrastructures but they did not consider spot instances, which is one of the main differences with respect to our work.

It is worth noting that, from the related works found, most of them have been proposed for workflows considering task deadlines or budget constraint but without considering spot instances. Besides, another crucial distinction is that in none of the surveyed works the authors have considered to reduce the impact of OOB errors as part of the optimization process when using spot instances, and only in three works [7, 26, 27] the authors have proposed to minimize the makespan, rendering difficult their applicability to execute scientific workflows in clouds infrastructures to support rapid domain-specific "in vitro" experimentation. Concretely, in the present paper the objectives are to minimize the makespan, monetary cost and the failure probability when different types of spot instances and bid prices are considered.

\section{Conclusions and Future Work}

Due to the complexity of scientific and engineering processes, scientific and engineering workflows have become increasingly challenging from a computational standpoint. Then, such workflows are often required to be executed in a distributed high-performance computing environment such as a cloud. A cloud combines the customization of instances with scalability and resource sharing capabilities. Moreover, clouds allow users the acquisition of instances under a pay-per-use scheme and where the prices differ according to the type of instance acquired and the pricing model of the cloud provider. Concretely, there are two pricing models through which instances can be acquired, i.e. on-demand and spot instances.

In this work we have proposed a new autoscaler for scientific and engineering workflows denominated CMI. This autoscaler exploits the elasticity potential of cloud infrastructures by approximating the right amount of on-demand and spot instances. To cope with the dynamically changing computing demands of workflows during execution, CMI periodically adjusts the number of instances of each type and determines the adequate bid prices to acquire spot instances aiming at minimizing makespan, monetary cost and the potential impact of task failures. These optimization problems are solved by using the popular meta-heuristic NSGA-II. A sensitivity analysis of NSGA-II was performed to determine the best hyperparameter set for each workflow application, together with detailed guidelines regarding how this analysis could be conducted for other workflows.

Simulation experiments on 4 large-scale scientific and engineering workflows indicate that CMI outperforms a closely-related state-of-the-art heuristic autoscaling strategy called SIAA in terms of the $L_{2}$-norm 
of makespan and cost. In this work, in order to perform a rigorous comparative study, we set up SIAA with 55 different configurations involving different combinations between on-demand and spot instances and different bidding strategies that trade-off cost and reliability. Statistical tests show that CMI outperforms SIAA in 41 of 44 cases, with statistically significant improvements regarding the $L_{2}$-norm metric. In the remaining 3 cases differences are not statistically significant. We believe these results are highly encouraging and of practical relevance.

This work can be extended by deepening the study of spot prices and failure probabilities due to OOB errors. Obtaining a more informative model of such errors that takes into account the expected duration of tasks will potentially help in the reduction of failures as bid prices could be better fitted to the expected duration of tasks. We expect that such a model could lead to improving the overall performance of CMI.

Another aspect that deserves special attention is to explore the ideas exposed in this article in the context of other evolutionary meta-heuristic algorithms such as NSGA-III, $\epsilon$-MOEA, MOEA/D, which are more suitable for solving many-objective problems than NSGA-II. Performing an extensive comparison of algorithms robustness under different settings and varieties of workflows will provide a deeper understanding of the characteristics of the problem and their solutions.

Finally, we are interested on extending the mathematical model of the autoscaling problem to consider data storage cost and storage access times. Designing a data-aware formulation for the autoscaling problem is fundamental for running today's data-intensive scientific workflows, and in this way not only supporting CPU-intensive workflows. In such context the occurrence of OOB errors might seriously handicap makespan derived from to the need of re-transmissions inside the cloud of huge amounts of data but also might critically increase execution costs due to extra use of storage/network resources, which must be rented in pay-per-use clouds.

\section{Reproducibility}

Workflow descriptions are available on-line through the Pegasus WorkflowGenerator 11 . Spot prices data used in this experiment are publicly available [20]. Experiments performed in this study were run on an Intel Core i5 computer running Ubuntu Desktop 16.0 and Java version 1.9. Implementation of the NSGA-II algorithm and the tools for the sensitivity analysis were provided by the MOEA Framework ${ }^{12}$ version 2.12. Simulations were performed using the CloudSim ${ }^{13}$ simulator version 3.0. We used implementation of the Mann-Whitney U test incorporated in Apache Commons Math ${ }^{14}$ version 3.3 for evaluating the statistical significance of our results. Visualizations were generated using Tableau Publiq version 2018.3 and ggplot $2{ }^{16}$ version 2.2.1.

\section{Acknowledgments}

This research is supported by the ANPCyT projects No. PICT-2012-2731, PICT-2014-1430 and PICT2015-1435, and by the SIIP-UNCuyo project No. M041. This research has been partially funded by the Spanish Ministry of Science and Innovation and FEDER under contract TIN2017-88213-R and the network of smart cities CI-RTI (TIN2016-81766-REDT).

\section{References}

[1] L. Ramakrishnan and D. Gannon. A survey of distributed workflow characteristics and resource requirements. Technical report, Indiana University Technical Report TR671, 2008.

\footnotetext{
${ }^{11}$ WorkflowGenerator: http://confluence.pegasus.isi.edu/display/pegasus/WorkflowGenerator

${ }^{12}$ MOEA Framework: http://moeaframework.org/

${ }^{13}$ CloudSim: http://www.cloudbus.org/cloudsim/

${ }^{14}$ Commons-Math: http://commons.apache.org/proper/commons-math/

${ }^{15}$ Tableau Public: http://public.tableau.com

${ }^{16}$ ggplot2: http://ggplot2.tidyverse.org
} 
[2] P. Maechling, E. Deelman, L. Zhao, R. Graves, G. Mehta, N. Gupta, J. Mehringer, C. Kesselman, S. Callaghan, D. Okaya, H. Francoeur, V. Gupta, Y. Cui, K. Vahi, T. Jordan, and E. Field. SCEC CyberShake workflows-automating probabilistic seismic hazard analysis calculations. In I.J. Taylor, E. Deelman, D.B. Gannon, and M. Shields, editors, Workflows for e-Science: Scientific Workflows for Grids, pages 143-163. Springer London, 2007.

[3] David A. Monge, Yisel Garí, Cristian Mateos, and Carlos García Garino. Autoscaling scientific workflows on the cloud by combining on-demand and spot instances. Computer Systems Science and Engineering, 32(4, Special issue: Elastic Data Management in Cloud Systems):291-306, July 2017.

[4] David A. Monge, Elina Pacini, Cristian Mateos, and Carlos García Garino. Meta-heuristic based autoscaling of cloud-based parameter sweep experiments with unreliable virtual machines instances. Computers \& Electrical Engineering, 69:364-377, 2018.

[5] E. Pacini, C. Mateos, and C. García Garino. Balancing throughput and response time in online scientific clouds via ant colony optimization. Advances in Engineering Software, 84:31-47, 2015.

[6] Z. Cai, X. Li, R. Ruiz, and Q. Li. A delay-based dynamic scheduling algorithm for bag-of-task workflows with stochastic task execution times in clouds. Future Generation Computer Systems, 71:57-72, 2017.

[7] E. De Coninck, T. Verbelen, B. Vankeirsbilck, S. Bohez, P. Simoens, and B. Dhoedt. Dynamic autoscaling and scheduling of deadline constrained service workloads on iaas clouds. Journal of Systems and Software, 118:101-114, 2016.

[8] J. Li, S. Su, X. Cheng, M. Song, L. Ma, and J. Wang. Cost-efficient coordinated scheduling for leasing cloud resources on hybrid workloads. Parallel Computing, 44:1-17, 2015.

[9] G. Juve, A. Chervenak, E. Deelman, S. Bharathi, G. Mehta, and K. Vahi. Characterizing and profiling scientific workflows. Future Generation Computer Systems, 29(3):682-692, 2013.

[10] M. Mao and M. Humphrey. Scaling and scheduling to maximize application performance within budget constraints in cloud workflows. In 27th International Symposium on Parallel \& Distributed Processing, pages 67-78. IEEE Computer Socienty, 2013.

[11] V. Turchenko, V. Shults, I. Turchenko, R.M. Wallace, M. Sheikhalishahi, J.L. Vazquez-Poletti, and L. Grandinetti. Spot price prediction for cloud computing using neural networks. International Journal of Computing, 12(4):348-359, 2013.

[12] David A. Monge, Matěj Holec, Filip Železný, and Carlos García Garino. Ensemble learning of runtime prediction models for gene-expression analysis workflows. Cluster Computing, pages 1-13, 2015.

[13] K. Deb, A. Pratap, S. Agarwal, and T. Meyarivan. A fast and elitist multiobjective genetic algorithm: Nsga-ii. IEEE Transactions on Evolutionary Computation, 6(2):182-197, Apr 2002.

[14] F.G. Montoya, F. Manzano-Agugliaro, S. López-Márquez, Q. Hernández-Escobedo, and C. Gil. Wind turbine selection for wind farm layout using multi-objective evolutionary algorithms. Expert Systems with Applications, 41(15):6585-6595, 2014.

[15] Mouna Rekik, Khouloud Boukadi, and Han $\tilde{A}^{a}$ ne Ben-Abdallah. An end-to-end framework for context-aware business process outsourcing to the cloud. Computers \& Electrical Engineering. , 2017. In press.

[16] W. Yu, B. Li, H. Jia, M. Zhang, and D. Wang. Application of multi-objective genetic algorithm to optimize energy efficiency and thermal comfort in building design. Energy and Buildings, 88:135143, 2015. 
[17] Andrea Saltelli, Marco Ratto, Terry Andres, Francesca Campolongo, Jessica Cariboni, Debora Gatelli, Michaela Saisana, and Stefano Tarantola. Global sensitivity analysis: the primer. John Wiley \& Sons, 2008.

[18] T Tang, P Reed, T Wagener, and K Van Werkhoven. Comparing sensitivity analysis methods to advance lumped watershed model identification and evaluation. Hydrology and Earth System Sciences Discussions, 3(6):3333-3395, 2006.

[19] R. Calheiros, R. Ranjan, A. Beloglazov, C. De Rose, and R. Buyya. Cloudsim: A toolkit for modeling and simulation of Cloud Computing environments and evaluation of resource provisioning algorithms. Software: Practice \& Experience, 41(1):23-50, 2011.

[20] David A. Monge. Amazon web services (AWS) spot prices data 2016. Mendeley Data, Nov 2018. , v1, http://dx.doi.org/10.17632/zcnp5xwvz6.1.

[21] H. B. Mann and D. R. Whitney. On a test of whether one of two random variables is stochastically larger than the other. The Annals of Mathematical Statistics, 18(1):50-60, 1947.

[22] M.A. Netto, C. Cardonha, R.L. Cunha, and M.D. Assuncao. Evaluating auto-scaling strategies for cloud computing environments. In 22nd International Symposium on Modelling, Analysis Simulation of Computer and Telecommunication Systems, pages 187-196. IEEE Computer Socienty, 2014.

[23] S. Lu, X. Li, L. Wang, H. Kasim, H. Palit, T. Hung, E.F.T. Legara, and G. Lee. A dynamic hybrid resource provisioning approach for running large-scale computational applications on cloud spot and on-demand instances. In 2013 International Conference on Parallel and Distributed Systems, pages 657-662, 2013.

[24] Y. Ding, G. Yao, and K. Hao. Fault-tolerant elastic scheduling algorithm for workflow in cloud systems. Information Sciences, 393:47-65, 2017.

[25] M. Mao and M. Humphrey. Auto-scaling to minimize cost and meet application deadlines in cloud workflows. In 2011 International Conference for High Performance Computing, Networking, Storage and Analysis (SC), pages 1-12, 2011.

[26] C. Qu, R.N. Calheiros, and R. Buyya. A reliable and cost-efficient auto-scaling system for web applications using heterogeneous spot instances. Journal of Network and Computer Applications, 65:167-180, 2016.

[27] D. Poola, S.K. Garg, R. Buyya, Y. Yang, and K. Ramamohanarao. Robust scheduling of scientific workflows with deadline and budget constraints in clouds. In 28th International Conference on Advanced Information Networking and Applications, pages 858-865. IEEE Computer Socienty, 2014. 